\title{
Plans for Blue Book 2010
}

\section{SUMMARY}

The Blue Book 2010 (BB10) and Pink Book 2010 (PB10) will be published at the end of June this year. In line with Office for National Statistics (ONS) policy, this article is intended to inform users about the content of these publications, including forthcoming changes.

\section{Scope of Blue Book 2010}

( NS has been working for some time on system changes to improve the quality of the National Accounts.

The current development programme (Effective National Accounts and Blue Book to measure the economy - 'ENABLE') aims to complete by March 2011 the integration of the National Accounts production systems, and their development and migration to a new IT platform. The Blue Book in 2010 will therefore be run on existing systems, using largely existing methodology. In addition, the relocation of National Accounts work from London will be largely complete by summer 2010 .

The scope and timing of the Blue Book in 2010 will be:

- the Quarterly National Accounts consistent with the 2010 Blue Book dataset will be published on 30 June 2010

- the reference year will be $2006=100$

- the last base year for the chained volume measure of Gross Domestic Product (GDP) will be 2006

- revisions to GDP will be taken on for 2006 and subsequent years

- there will be no revisions to GDP before 2006

- all 'industry' outputs will be classified according to the Standard Industrial Classification 2003

- in cases where Average Earnings Indexes are used for deflation, these will be replaced by the more conceptually appropriate Average Weekly Earnings indexes
- Retail Price Indexes will continue to be used for the purposes of deflation (that is adjusting current price estimates of GDP onto a 'volume' basis). It is expected that this will change in the Blue Book 2011 (see the section on 'Future developments' later in this article)

\section{Methodology changes}

One significant methodological change, which will be introduced during 2010 , relates to the measurement of the activity of the Insurance industry. The methodology used currently is based on the conventions set out in the European System of Accounts 1979 (ESA79). Work is now well advanced to bring this into line with ESA95. This new Insurance methodology will be used as the basis of the estimates provided to the European Statistical Office (Eurostat) within the UK's Gross National Inventory (GNI) return in September 2010. (This return forms the basis of the UK's contribution to European funding under what is known as 'the fourth resource'). Revisions to GDP from this change will affect all periods from 1995. The estimates will be published in the UK, but it is not planned to implement the change fully in the published UK National Accounts until Blue Book 2011, when it will affect both current price and volume estimates of GDP.

\section{Scope of Blue Book 2011}

As mentioned earlier, the ENABLE programme is on track to complete, as planned, in March 2011. The programme 
will deliver an integrated system for the production of estimates of GDP and the Sector Accounts. This integration will improve considerably the compilation process used for National Accounts, and is designed to support greater analysis of the dataset, improve the consistency of the estimates and the transparency of the processes used to align the three different measures of GDP (Production, Expenditure and Income). This will be a significant step forward for the quality of the UK National Accounts.

Initial plans for the scope and content of the Blue Book in 2011 include:

- the reference year will be $2008=100$

- the last base year for the chained volume measure of GDP will be 2008

- the new methodology for measuring the activity of the Insurance industry (described above) will be introduced for the volume measure of GDP

- Public Sector Finance statistics will be made more consistent with National Accounts estimates

- improvements to Financial Services statistics, developed under a related programme, will be introduced

- where Retail Price Indexes (RPIs) are used currently for the purposes of deflation (that is adjusting current price estimates of GDP onto a 'volume' basis) the Consumer Prices Indices will be used instead (this provides a conceptually more relevant basis for deflation)

- in line with European standards, all 'industry' outputs will be classified on the new Standard Industrial Classification 2007

A related development will be the production of current price Input Output Analytical (I/O) tables. These were last produced in the early 2000s for the year 1995. New tables, for 2005 , are now being complied and planned for publication in 2011.

\section{Future developments}

Over the next 2-3 years ONS plans to introduce a number of significant improvements to the systems, methods and analysis supporting core National Accounts outputs. As already noted, the ENABLE programme will provide an integrated and robust basis for producing the Quarterly National Accounts and the annual Blue Book from September 2011. The simplifications and integration of the National Accounts processes planned within the programme will also produce an environment to 'enable' future developments.

Beyond ENABLE, work is already underway on the next phases of the development of the UK National Accounts. Within this there are two broadly distinct work programmes: a set of medium term goals planned for implementation by 2014, and some longer term aspirations. The medium term goals include the preparation for the transition to the new European System of Accounts 2010 (yet to be finalised), which the UK will implement in line with European regulation in 2014.
The key changes here include the treatment as capital of Research and Development activities, improvements to the treatment of pension statistics, treatment of goods sent abroad for processing and the delineation between market/non-market activities.

Consistent with the longer term aspirations for the development of the UK National Accounts, ONS also plans further methodological research. Some additional developments being considered in the next few years include:

- further research into the potential for balancing the three measures of GDP in constant price (volume) terms, in contrast to the existing approach which involves balancing nominal, or current price, estimates. Initially, this may be just for annual estimates, but ONS will investigate the feasibility of producing quarterly constant price Supply and Use tables, which would form the basis of the official estimates of GDP

- improvements in the measurement of Financial Services

- further development of the so-called 'satellite' accounts (including accounts for Research and Development and the Environment)

- research into wider measures of wellbeing; and

- the development of a labour accounting framework

囚 시r@ons.gov.uk 